\title{
Exploiting Virtual Elasticity of Manufacturing Systems to Respect OTD-Part 2: Post-Optimality Conditions for the Cases of Ergodic and Non-Ergodic Order Rate with Deterministic Product-Mix
}

\author{
Bruno G. Rüttimann1, Martin T. Stöckli² \\ ${ }^{1}$ ETH Zürich IWF, Zurich, Switzerland \\ ${ }^{2}$ Inspire AG, Zurich, Switzerland \\ Email: bruno.ruettimann@inspire.ethz.ch, stoeckli@inspire.ethz.ch
}

How to cite this paper: Rüttimann, B.G. and Stöckli, M.T. (2021) Exploiting Virtual Elasticity of Manufacturing Systems to Respect OTD-Part 2: Post-Optimality Conditions for the Cases of Ergodic and Non-Ergodic Order Rate with Deterministic Product-Mix. American Journal of $O p$ erations Research, 11, 141-165.

https://doi.org/10.4236/ajor.2021.113009

Received: March 5, 2021

Accepted: May 11, 2021

Published: May 14, 2021

Copyright $\odot 2021$ by author(s) and Scientific Research Publishing Inc. This work is licensed under the Creative Commons Attribution International License (CC BY 4.0).

http://creativecommons.org/licenses/by/4.0/

\begin{abstract}
Respecting the on-time delivery (OTD) for manufacturing orders is mandatory. However, for non-JIT Batch \& Queue Push-manufacturing systems, the compliance of OTD is not intrinsically guaranteed. As an OTD related manufacturing theory is largely missing it is crucial to understand and formalize the necessary conditions of OTD compliance for complex production environments for maximum exploitation of the production capacity. This paper evaluates the conditions of post-optimality while being OTD compliant for production systems, which are characterized by stochastic order rate and a deterministic product-mix. Instead of applying discrete event simulation to explore the real case-by-case order scheduling optimization for OTD compliance, a Cartesian approach is followed. This enables to define theoretically the solution space of order backlog for OTD, which contributes to developing further manufacturing theory. At the base stands the recently defined new concept of virtual manufacturing elasticity by reducing lead-time to increase virtually production capacity. The result has led to defining additional two corollaries to the OTD theorem, which sets up basic OTD theory. Apart from defining the post-optimal requirements to guarantee for orders at least a weak solution for OTD compliance, this paper reveals that for a deterministic product-mix a non-ergodic order arrival rate can be rescheduled into an ergodic order input rate to the shopfloor if the virtual elasticity $\Delta T$ is large enough, hence the importance of having fast and flexible production lines.
\end{abstract}




\section{Keywords}

On-Time-Delivery, Lean Manufacturing, Industry 4.0, Arrival Rate, Non-Ergodic Process, Virtual Elasticity, Normed Exit Rate, Ergodicity

\section{Introduction and Approach to the Problem}

As the fulfillment of customer requirements for product quality, the on-time delivery (OTD), which is the punctual delivery of manufactured products, is essential for a customer-satisfaction sustainable business. The OTD requirement can be considered as fulfilled, if deliveries match the expected delivery time $(E D T)$ - the jointly agreed date of delivery. Whereas a widely used key metrics to calculate OTD exists, which is the ratio of goods delivered to customers on time related to the total goods deliveries, the necessary and sufficient conditions for OTD compliance have not yet been a topic of mainstream manufacturing theory. Indeed, this apparently trivial, but important objective has rarely been researched. The reason is simple: progressing simulation of complex manufacturing systems has prevented the development of such a theory. With a series of papers, we intend to bridge this gap.

This paper is a generalization of a previous paper [1], which examined the post-optimality conditions to comply with the OTD requirement for time-bound order rate $(O R)$ above production capacity with deterministic or mainly ergodic order-arrival characteristic for a single mono-product manufacturing. A manufacturing theory textbook evoking the topic of OTD is [2]. It is the first textbook approaching systematically the topic of OTD from a theoretic, law-based view defining the necessary and sufficient conditions for OTD in a mono-product manufacturing system. With the present paper we extend the problem setting by developing the post-optimality conditions for OTD of a multi-product manufacturing system showing a predominant non-ergodic stochastic arrival $O R$ characteristic, however, with a deterministic product-mix. This topic is of high interest in view of forthcoming graph-based and artificial intelligence (AI)-controlled new cyber physical production systems (CPPS). This paper analyses and allows fully understanding the preconditions for OTD of a highly non-ergodic process within a non-deterministic product-mix production environment. Indeed, the OTD topic has not yet been examined analytically, but the solution is usually hands-on simulated at the occasion of real order scheduling.

In [1] were expanded the necessary and sufficient conditions for OTD, basic conditions defined with the OTD theorem in [2], with post-optimality conditions enouncing two additional corollaries. It reveals that a temporary (during $\Delta t)$ higher $O R$ than the exit rate $(E R)$ of a production system in a fix capacity regime can be backlog-buffered and rescheduled according to the difference $\Delta T$ of expected delivery time (EDT) and the process lead time $(P L T)$. Due to this, the process is OTD compliant. The $\Delta T$ is the virtual manufacturing elasticity leading 
to increased capacity, which has been defined in [1]. The increased capacity is only virtually increased and not physically. Physical nominal production capacity ER is still given by the cycle time $C T_{b}$ at the bottleneck. The virtual elasticity, however, allows supplying the customer order exceeding bottleneck-capacity still on time. This reveals the importance of fast lean manufacturing lines reducing PLT. Two cases were distinguished and have been solved for deterministic mono-product $O R$ (Equation (1)) and for stochastically variable ergodic $O R$ process (Equation (2)), with post-optimality of $x(t)$ for OTD

$$
\begin{gathered}
\left(\frac{x(t)}{\lambda}-1\right) \cdot \Delta t \leq f(\Delta T) \\
\int_{t_{1}}^{t_{2}}\left(\frac{x(\tau, t)}{\lambda}-1\right) \cdot \mathrm{d} t \leq f(\Delta T)
\end{gathered}
$$

where the variable $x(t)$ represents a deterministic, but time-bound variable OR and $x(\tau, t)$ represents a stochastic $O R$ with variable inter-arrival time $\tau$, the $\lambda$ represents the constant $E R$ which should be in line with the average $O R$. The findings of the paper also have led to enounce the Second Corollary to the Theorem of General Production Requirement (Corollary of Strong and Weak OTD Solutions) formulating the necessary and sufficient condition for a strong OTD solution for Equations (1) and (2) [1]:

\section{Second Corollary to the Theorem of General Production Requirements (Corollary of Strong and Weak OTD Solutions):}

Given is an order rate with generally random arrival character within a fixed-capacity regime. Necessary and sufficient condition for a strong OTD solution is the ergodic characteristic of a stochastic order process paired with symmetric distribution of order rates of the random OR variable and if they comply with the requirements of the OTD theorem. However, non-ergodic processes with erratic arrivals, or ergodic processes with asymmetric random distributions of order rate, constitute only a weak solution for OTD observance, observance depending on the specific circumstances. The circumstances are the distribution of arrivals, the sequence of arrivals, and timespan of non-randomness, as well as dependency on the virtual elasticity of the production system.

It is important to note that the problem of post-optimality does not represent a topic of interest in TPS-derived perfect lean just-in-time (JIT) production systems, because the product-mix and OR are deterministically known and therefore the manufacturing system can be conceived and implemented accordingly to meet the OTD requirement. This is not only important, but also interesting and shows the sophisticated functioning of the Toyota JIT production system. A rational and formalised scientifically founded description of the JIT production system, as well as basic manufacturing theorems, have been developed in [2] defining production-related laws and implementation principles. The JIT production system relies mainly on a make-to-stock production principle. TPS-derived 
lean JIT productions base on pull manufacturing principle with one piece transfer principle forming a perfect single piece flow (SPF) and shows an even $O R$, called takt rate $(T R)$ with ideally $S D[T R]=0$, which corresponds to a deterministic order regime. Furthermore, the Toyota JIT lean production system relies on a deterministic and predefined product-mix leading to ergodic-type of processes. In addition, manufacturing batches produced in multi-product manufacturing cells (mixed model) are standardized in equal timeslots called pitches to reduce Mura (unevenness), while the production-mix is alternated using Heijunka-box levelled scheduling. This allows having all products, but also all necessary intermediate components along the manufacturing sequence, ready to be assembled according to customization specification and finally supplied to the next workstation, or delivered to the customer, nearly instantaneously. Taiichi Ohno (Toyota), the father of the JIT production system, extended Henry Ford's mass production into a flexible production system. However, a perfect smooth running JIT system bases on a differentiated, but deterministic product-mix, which, nevertheless, can be flexibly called-off.

In this paper we will analyse the OTD compliance for a deterministic product-mix, which ideally should be manufactured, under ideal conditions, using a lean JIT-based pull manufacturing system and not a batch and queue (B \& Q) system. However, we will analyse the consequences to a traditional push B \& Q manufacturing system, without dealing immediately with stochastic product-mix variability of envisaged CPPS. This intermediate step of analysis allows understanding the requirements to pay attention for the omnipotent CPPS, Industry 4.0 manufacturing system characterized by non-ergodic order processes with stochastically variable product-mix. In the following, we will apply the same approach, which has already been used in the paper [1]. That paper mainly dealt with the elasticity topic of production systems, i.e. the insensitivity of cost-change to production volume change. In the present paper, we will not yet consider the flexibility topic, i.e. the insensitivity of cost-change to production mix variability, applied for OTD compliance. This in order not to explode the complexity of the topic to be modelled-it will be the topic of a next paper. Similar to the distinction of experimental and theoretic physics, in the following, we will not employ the usually applied discrete event simulation (DES) technique to gain insights, but continue to derive the development of the solution to the problem based on analytic law-based production theory, approach defined for the first time in the modern manufacturing theory textbook [2]. This scientific-theory approach bases on solid production theorems and corollaries related to manufacturing laws. Indeed, instead of exploring the behaviour heuristically by simulation, or even worse empirically by trial and error, a Cartesian-based approach allows understanding the intrinsic functioning of a production system to get a formalized description of the laws governing OTD.

This topic is highly innovative and not yet researched. The reason is linked to the lack of a mathematical definition of OTD, given only in 2017 with the OTD theorem [2], which states the necessary and sufficient condition to supply an 
order on time:

\section{Theorem of General Production Requirements (or OTD Theorem):}

The necessary and sufficient conditions to supply a customer with OTD, i.e. with the right quantity at the right time, is that first the capacity requirement and second the lead time requirement have to be satisfied simultaneously, independent of the applied transfer principle, i.e. SPF or B \& Q. The capacity requirement is given by the Corollary of Weak WIP Stationarity and the lead time requirement necessitates that $M L T$ plus $B W T$ is shorter than $E D T$.

We have reported here again the OTD theorem, because it is central to the present problem development and is generally one of the most important theorems for manufacturing on which further corollaries can be derived.

\section{A Little Bit More Theory and Preliminary Assumptions}

In the following, we analyse arrival processes of $O R$ cases with increased complexity and variability leading to non-ergodic processes. Analysing a manufacturing system, the full attention has to be put on the bottleneck. Remembering the Bottleneck Theorem for a mono-product manufacturing line, the bottleneck is the machine or, general speaking, the operation with the longest cycle time $(C T)$ determining the exit rate $(E R)$ of the production line. For a detailed examination of the concept of bottleneck and related theorem and corollaries, we refer to [2]. Due to the presence of a product-mix, requiring flexible production equipment, we cannot rely anymore on the Second Corollary to the Theorem of Throughput (Corollary of Bottleneck Time-invariance) valid for a mono-product manufacturing process. Indeed, a product-mix entails that the ER might change. Also the Theorem of Throughput (or Bottleneck Theorem) has to be extended and the concept of bottleneck-equipment has to be redefined when a mix of products are manufactured on shared equipment leading to the Theorem of Generalized Throughput (or WTT-Aggregated Bottleneck Theorem) [2]. This is also valid for the bottleneck cell within a complex inter-linked production of various manufacturing cells. To identify the bottleneck workstation, or bottleneck cell, of a mixed-production we have to introduce the concepts of workstation turnover time (WTT) and cell turnover time (CTT). The WTT is the time to produce once the whole mix and can be identified with $W T T_{m, K}$ where $m$ identifies the specific machine (workstation) and $K$ represents the products manufactured on the workstation. The $W T T_{m, K}$ is defined for the $m^{\text {th }}$ workstation with the product-mix of $K$ products in Equation (3) where the capital $K$ refers to the mix of products and the minor $k$ to the specific product

$$
W T T_{m \mid K}=\sum_{k}\left(S T_{m \mid k}+C T_{m \mid k} \cdot B_{k}\right)
$$

where $S T_{m, k}$ means set-up time of the workstation $m$ for the $k^{\text {th }}$ product and $C T_{m, k}$ is the cycle time of the $m^{\text {th }}$ workstation for the $k^{\text {th }}$ product and $B_{k}$ is the batch-size of the $k^{\text {th }}$ product. For a detailed examination of the concepts of WTT 
and $C T T$, we refer again to [2]. The introduction of $W T T$ is required for a deterministic product mix to identify the bottleneck workstation. In the case of non-deterministic product mix, the bottleneck does not only change dynamically (see the corollary reported hereafter), but the definition of WTT makes less sense, because of the difficulty to define the mix. For the definition and calculation of $C T T$ we refer to [2]. We have to anticipate that the concept of manufacturing cell makes mainly sense for standard or standard-like components of a defined mix. Indeed, all the products to be manufactured within the same cell have to follow a defined sequence of operations according to the Theorem of Cell Product Congruency (or Linear Dependency Theorem) or to the Corollary to the Theorem of Cell Product Congruency (Corollary of Imperfect Dependency) [2].

\section{Theorem of Cell Product Congruency (or Linear Dependency Theo- rem):}

Necessary condition to put similar but different products undergoing the same treatments/operations into the same manufacturing cell is that the cycle times $C T_{m k}$ elements of the cell matrix have to be linearly dependent.

A manufacturing cell or transfer line (TFL) constitutes a "productive capacity" suitable for LMHV (low mix high volume) production best represented by a flowshop organization. For high variable mix including one-offs (HMLV) a "technological competence" is required with variable production paths between the workstations (jobshop organization) such as envisaged by CPPS-organised shopfloor, workstations which have to be served by AGVs (Automatically Guided Vehicle). The concepts of WTT and CTT lead to the Theorem of Generalized Throughput (or WTT-aggregated Bottleneck Theorem) and, in the case of not observance of the Linear Dependency Theorem, to the First Corollary to the Theorem of Generalized Throughput (Corollary of Generalized Bottleneck Time-variance) [2]:

\section{Theorem of Generalized Throughput (or WTT-Aggregated Bottleneck Theorem):}

In a multi-product manufacturing cell (or transfer line) with cycle times $C T_{i \mid k}$ the workstation (or cell) with the longest $W T T_{i}$ (or $C T T$ ) determines the bottleneck workstation (or bottleneck cell) to supply a mix of products. Please note, the exit rate $E R$ of the cell is still given by the workstation with longest $C T_{i \mid k}$ processing a certain product.

and

First Corollary to the Theorem of Generalized Throughput (Corollary of Generalized Bottleneck Time-Variance):

Different than in mono-product cells (or line) where the bottleneck is time-invariant (under "ceteris paribus" conditions), in mixed-product manufacturing cells with high differences between the cycle times $C T_{i \mid k}$ of different products $k$, the operation $i$ for a product $k$ being the bottleneck 
may vary and the bottleneck being therefore time-variant according to the product $k$ in the cell.

Furthermore, a mixed-product manufacturing introduces an additional complication compared to a mono-product manufacturing. Indeed, the usually applied concept of process lead-time $(P L T)$ of a single product has to be substituted by the concept of manufacturing lead-time $(M L T)$ of an entire batch. The $M L T$ is the timespan when the first piece of the batch enters the manufacturing process and the last piece of the batch leaves the manufacturing process. As per the Theorem of Generalized Lead-Time (or Speed Theorem) the MLT depends on the transfer principle B \& Q or SPF. $M L T$ can be computed for a perfectly SPF balanced line with $m$ equal $C T_{k}$ by Equation (4); the $C T_{k}$ refers here to the specific product.

$$
M L T_{k}^{\mathrm{SPF}}=\left(B_{k}-1+m\right) \cdot C T_{k}
$$

For the case of a production line applying an n-piece transfer principle leading to an n-piece flow (nPF) consult [2] for the equations to calculate the $M L T_{\mathrm{nPF}}$ variant. A complete derivation of equations and transformations between $P L T$ and $M L T$ for the different transfer principles is given in [2]; we repeat here only important conclusions. Equation (4) shows that the bigger the batch $B_{k}$ of product $k$ is and the more workstations or machines $m$ have to be used to manufacture the product, the bigger becomes the difference between MLT and PLT, hence the fundamental importance to use the $M L T$ concept of lead-time in this case. In addition, $M L T_{\mathrm{SPF}}$ is always shorter than $M L T_{\mathrm{B} \& \mathrm{Q}}=B_{k} \cdot m \cdot C T_{k}$ manufacturing lead-time of a B \& Q production principle, law expressed by the Main Theorem of Production Time (or SPF Dominance Theorem) and its corollary and lemma [2]. Stressing the importance of delivering the entire batch completely is represented also by the enlarged concept of OTD stating "on-time-in-full” deliveries (OTIF). This entails that we cannot use the OTD equation system used in [1], but we have to use equation system 5 substituting PLT with $M L T$ derived from the Theorem of General Production Requirements (or OTD Theorem) giving the necessary and sufficient conditions for OTD compliance.

$$
\begin{aligned}
& \text { for }: S D[O R]>0 \\
& \left\{\begin{array}{l}
\forall i: \inf \left\{E R_{i}\right\}>E[O R] \\
Z: B W T_{Z}+M L T_{Z} \leq E D T
\end{array}\right.
\end{aligned}
$$

The index $Z$ in Equation (5b) indicates the order entry point of the process from which the customer perceives the lead-time, called customer visible time (CVT). Therefore, the whole PLT (or $M L T$ ) starting from the raw materials might differ from the CVT. Typically, the entry point $Z$ could be a customization operation buffered with up-stream standard components supermarkets.

Another complication in the analysis of a realistic order situation compared to the simplistic mono-product manufacturing analysed in [1] is that in a multi-product environment each order refers to different products with different 
order sizes. Therefore, we have to add to the arriving OR distribution $X_{k}$ also the customer-related order information writing according to Equation (6)

$$
\Omega_{k}:=\left(X_{k}, B_{k}, E D T_{k}\right)
$$

Each product order $\Omega_{k}$ is characterized by a 3-tupel (or triple) where the index $k$ refers to the specific product $k$. We can therefore claim that the entity "order" $\Omega_{k}$ belongs to a 3-dimensional vector-space of complex data-range defined for non-negative numbers and distributions. Indeed, associated with each order $\Omega_{k}$ is always the order quantity $B_{k}$ of the commercial order as well as an associated expected delivery time $\left(E D T_{k}\right)$. The commercial order might be split in the case of lean JIT manufacturing in n sub-batches of size $B_{k} / n$. Indeed, the Theorem of Lean Batch Sizing and its corollary, the Corollary of Cycle Time of a Mixed-Product Workstation, give the necessary conditions to assure the availability and timely replenishment of all products. However, we will assume to produce the ordered quantity in full as requested by classic customer orders (OTIF) with an entire batch transfer principle if not specified differently. This corresponds also to the traditional way, how Western companies produce and supply customers. If the $O R$ is non-deterministic, to model the performance we have to add additional information to the $O R$ variable $X$ about the distribution of the inter-arrival time $\tau$ and the potential verification of non-random distribution represented by $t$ leading to non-ergodic processes. The distribution of $O R$ given by $X(1 / \tau, t)$ or better the respective Markovian time-dimension of inter-arrival time $X^{-1}(\tau, t)$ represents the time-dimension of order arrival process. The presence of the index $k$ in $X_{k}^{-1}(\tau, t)$ refers to the specific product $k$, the absence refers to the general order entry load without considering which product arrives. In this paper, we prefer to use the capital Greek symbol $X(\tau, t)$ over the Roman notation $x(\tau, t)$ used in the former paper (part 1) for single piece order to indicate a batch-dimension order-entry with product-mix. The order entry rate $O R_{k}$ has to be transformed into a shopfloor input rate $I R_{k}$. If the release sequence of orders $\Omega_{k}$ is not FIFO but rescheduled, we use the symbol $\chi_{k}^{-1}(\tau)$ to indicate the inter-scheduling time (i.e. the time between the product $k$ is produced again) of the inter-arrival time $X_{k}^{-1}(\tau, t)$, else we keep the arrival notation. We will perform also a brief comparison between the here analyzed B \& $\mathrm{Q}$ behavior and the state-of-the-art lean JIT system, which is the ideal manufacturing system for such type of order and production regimes. We indicate with $(\tau)$ a stochastic (ergodic) and with $(\tau, t)$ a stochastic, but non-ergodic variable. The deterministic, stochastic, or non-ergodic nature of the scheduled variable is, however, often indicated generically with $(\tau, t)$ and needs a case by case consideration (Figure 1).

Interesting is the congruency of Markovian time-dimension of scheduled $I R$, i.e. the order inter-scheduling time $\chi_{k}^{-1}(\tau)$ with the product interval time (PIT) of Kanban-controlled and Heijunka pitch-levelled manufacturing cells [2]. We can already now anticipate that $\chi_{k}^{-1}(\tau, t)$ is the stochastic interpretation of 


\begin{tabular}{|c|c|}
\hline B\&Q: "on-time-in-full" (push) & lean-TPS:" just-in-time" (pull) \\
\hline order-arrival: $O R_{k} \rightarrow$ shopfloor: $I R_{k}$ & shopfloor:IR $R_{k} \leftarrow$ order-arrival $: T R_{k}$ \\
\hline 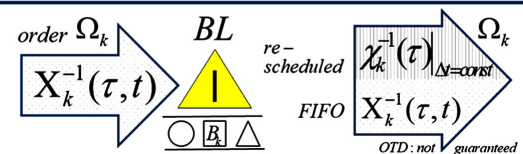 & 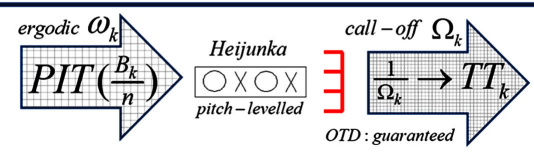 \\
\hline
\end{tabular}

Figure 1. Schematic comparison between push OTIF-delivery and pull JIT-production. The lean TPS comparison is shown to clarify the alternative possibility of a pull order-handling in deterministic product-mix environment compared to a classic B \& $\mathrm{Q}$ push.

deterministic $P I T_{k}$ PIT and $W T T$ together used to size Kanban supermarkets. However, we have to precise that $\chi_{k}^{-1}(\tau, t)$ is an external market-driven variable from $X_{k}^{-1}(\tau, t)$ whereas the PIT is an internally defined and adapted production variable (usually constant) used to replenish timely the supermarkets based on external TR-based call-offs. Furthermore, related to the single product-pieces, manufacturing is a deterministically resulting consequence of scheduling the single pieces of the batch considering the applied work shift regime, which translates into a takt-time $T T$; we can therefore write $T T_{k}$

In Figure 2 it is shown that the $P_{I T_{A}}$ of product $\mathrm{A}$ has a double reorder frequency than PIT of products B and C. In lean JIT manufacturing systems PIT should be a multiple of the pitch, hence the interest to reduce pitch. The pitch is a standard time-slot associated to a standard quantity (internal batch-size adapted to a manageable logistic transportation bin such as a pallet or another defined pack-out quantity). Note that pitch comprises set-up and processing time. Associated to the Theorem of Generalized Throughput for a Kanban-managed manufacturing cell is the Second Corollary to the Theorem of Generalized Throughput (Corollary of Frequency) and the Theorem of Lean Batch Sizing (or Optimal Batch Theorem) paraphrasing the driving variables of the manufacturing cell to replenish the supermarket on time. These facts show that lean manufacturing intends to bring non-ergodic order processes to deterministic production processes reducing by that complexity, facilitating or even eliminating production planning and allowing even a Cartesian-based description of production theory [2]. This shows that in presence of a deterministic mix the lean JIT pull manufacturing system should be preferred over a B \& Q push manufacturing system, at least for the part of the production mix, which is deterministic.

To each order $\Omega_{k}$ is unequivocally associated with a specific characteristic of the product $k$, which is the process mapping reflecting the required sequence of operations to manufacture the product. The sum of cycle times $C T$ of non-necessarily ordered sequence represents its work content (WC), which can be either touch-time (manually) or processing-time (machining), in any case it is the Muda-free value-add time-content of the product without waiting or transportation time.

$$
\Omega_{k} \rightarrow \forall k: \exists W C_{k}
$$




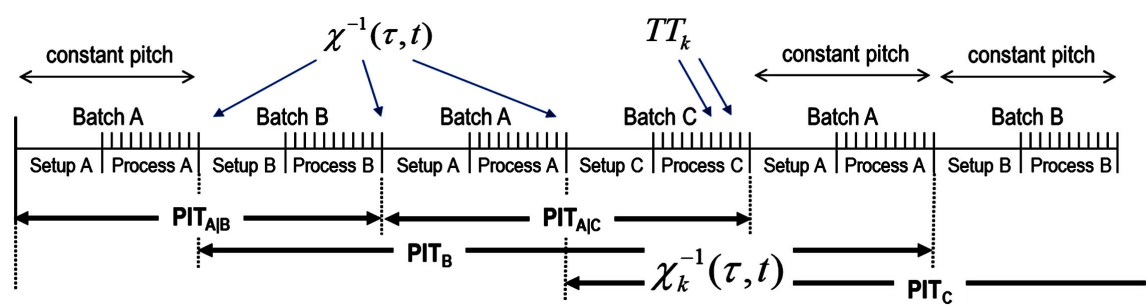

Figure 2. $\operatorname{PIT}_{\mathrm{k}}$ and $\chi_{k}^{-1}(\tau, t)$ congruency as well as respectively batch-indifferent constant pitch and $\chi^{-1}(\tau, t)$ congruency as well as product-specific takt-time $T T_{k}$, which may be different from $T T_{k+1}$ of another product. The figure shows the case of a pitch-leveled Heijunka-box scheduling for JIT manufacturing (adapted from [2]).

It is obvious that the WC can vary from product to product. We will simplify the problem by not allowing customization of product $k$ so that $W C_{k}$ is a deterministic and time-invariant constant for each product. In such deterministic cases we can map production as a deterministic matrix representing the input for master capacity mapping of production for a defined product-mix (Equation (7)).

$$
P=\left[C T_{m k}\right]=\left[\begin{array}{cccc}
C T_{11} & C T_{12} & \cdots & C T_{1 k} \\
C T_{21} & C T_{22} & \cdots & C T_{2 k} \\
\vdots & \vdots & \ddots & \vdots \\
C T_{m 1} & C T_{m 2} & \cdots & C T_{m k}
\end{array}\right]
$$

The rows of the production matrix $P$ in Equation (7) represent the $m$ machines (or generally the operations to be performed) and the columns the $k$ products forming a matrix of non-necessarily quadratic order with dimension $m \times k$ of unitary specific $C T$ representing the base to calculate the resource absorption. Therefore, the specific work content $W C_{k}$ of each product $k$ corresponds to the sum of the $C T$ of the column-vector of the matrix $\left[C T_{m k}\right]$, i.e.

$$
W C_{k}=\sum_{m} C T_{m k}
$$

Please note that in the matrix represented in Equation (7) it may happen that some $C T_{l i}=0$, where $1 \leq l \leq m$ and $1 \leq i \leq k$. Matrices according to Equation (7) form the base of Linear Programming problems of the type $A \cdot x \leq b$ to identify the optimal production-mix $x^{*}$ to maximize margin contribution function $z=c^{T} \cdot x$. However, such "ex ante" optimization techniques, although delivering Pareto-efficient solutions, are inferior to "live" operational lean JIT manufacturing systems [3]. Indeed, the optimal "ex-ante" defined mix cannot be reproduced in reality. However, lean JIT production allows reacting to the instantly requested real product-mix.

If there is a modern cellular-organized manufacturing system, i.e. we have a complex manufacturing system with multiple manufacturing cells interconnected (semantically-correct speaking they are not interconnected, but de-coupled, because linked via Kanban-managed supermarkets) the matrix of Equation (7) can be split into several matrices of lower order (Equation (8)). 


$$
C_{j}=\left[c t_{m-l \mid k-i}\right]_{j}=\left[\begin{array}{ccc}
c t_{11} & \cdots & c t_{1 k-i} \\
\vdots & \ddots & \vdots \\
c t_{m-l 1} & \cdots & c t_{m-l \mid k-i}
\end{array}\right]_{j}
$$

The different cells $j$ may comprise the same type of machines $m$ or product-specific different ones. The different matrix $C_{j}$ can even have the same products $k$ if the cells $j$ are manufacturing the product in components, which then are assembled in a separate cell.

In such cases of complex manufacturing the concept of bottleneck equipment computed with $W T T_{m}$ for each machine m of each cell $j$ has to be widened defining the cell turnover time $\left(C T T_{j}\right)$ for each cell $j$ identifying the bottleneck cell of production [2]. The splitting of Equation (7) in several new matrix $C_{j}$ for each cell $j$ (Equation (8)) usually of lower order $(m-l, k-i)$, where the matrix order (row and column dimensions) have not to be equal, can logically be represented by

$$
\left[C T_{m k}\right]:=\left\{\bigcup_{j}\left[C t_{m-l \mid k-i}\right]_{j} \mid \forall j: l_{j}<m_{j}, i_{j}<k_{j}\right\}
$$

Equation (8) applies ideally for such products $k$ if the $C T_{m k}$ of these products in Equation (7) are linearly dependent, then such similar products $k$ can be put into the same manufacturing cell represented by Equation (8). These products obey to the before mentioned Theorem of Cell Product Congruency (or Linear Dependency Theorem) or to the Corollary to the Theorem of Cell Product Congruency (Corollary of Imperfect Dependency). Therefore, the big difference regarding matrix of Equations (7) and (8) between lean JIT Kanban-managed cell and traditional cells consist of $C T$-affine products and non-affine $C T$ products. Indeed, for lean JIT manufacturing only products with linearly dependent CT of the process are grouped into the same cell, forming matrix of Equation (8). The linear dependency is not necessary for traditional B \& Q manufacturing cell, which may constitute a sort of mini jobshop organization where the different machines are grouped into a shopfloor layout improperly named cell (improper or fake cell), where the Corollary of Generalized Bottleneck Time-Variance applies. We could call such improper cells pseudo-cell-type manufacturing, but not cellular manufacturing. Therefore, to name a layout a cell, the Theorem of Cell Product Congruency should apply. A cellular manufacturing allows implementing a JIT manufacturing system. It is not important whether or not such cells are operated manually or implemented as automatic FMS (Flexible Manufacturing System).

In the following, we will solve the problem of OTD regarding a single cell $j$ according to Equation (8). Not only for JIT-managed production systems Equation (8) might be relevant; also for traditional B \& Q manufacturing systems Equation (8) applies. However, although considering non-lean JIT manufacturing systems, we will base the following considerations on lean JIT-derived principles [2] applied to non-Kanban supermarket decoupled cell-type manufacturing. 
In lean JIT manufacturing, the necessary number of equipment or workstations as well as number of required blue-collar operators to match with the market-imposed takt-time (TT) in order to be potentially on-time can be computed as follows

$$
m_{k}=\frac{W C_{k}}{T T_{k}}
$$

Equation (9) computes the necessary capacity to be able to face the imposed demand given by the $T T$, i.e. $T R$, where ideally $S D[T R]=0$. However, Equation (9) does not state if the number of equipment is tayloristically split (sequentialized) or $W C$-integrally multiplied (parallelized) in the manufacturing cells or TFL. Equation (9) allows to debottleneck and to balance the processing steps of the $W C$ applying the debottleneck principle. Please note that sequentialization will lead to change the order of the matrix $C_{j}=\left[c t_{m k}\right]$ with regard to the column dimension $\mathrm{m}$ (number of rows) with smaller cycle time $c t_{m k}$, but same $W C_{k}$ work content. For a mixed-product cell according to Equation (8) we can approximate Equation (9) with Equation (10) giving the number of operators (or respectively machines) and may correspond for Tayloristic split $W C$ to the dimension of the column-vector in matrix $\left[C T_{m k}\right]$ of Equation (7)

$$
m_{\text {cell }}=\frac{E[W C]}{E[T T]}
$$

The Theorem of Debottlenecking (or Dual Solution Theorem) and its four lemmas as well as the debottlenecking principles can help to adapt the necessary capacity to the required $O R$. Furthermore, with the de-bottleneck principles it is always possible to transform a non-balanced SPF into a balanced flow. Based on the Main Theorem of Production Time (or SPF Dominance Theorem) and its Corollary to the Main Theorem of Production Time (Corollary of Lead Time Limit) it is advisable to establish a perfectly balanced SPF and therefore we can enounce the following.

\section{Second Corollary to the Main Theorem of Production Time (Corollary of Balanced Line):}

Given is a non-balanced SPF. By applying the Tayloristic-sequentialization de-bottlenecking principle, it is possible to transform a non-balanced production line into a perfectly balanced line with equal cycle times.

Nevertheless, it has to be noted, this corollary is subject to the property of sub-additivity, i.e. it might result $\left\|\left(C T_{1}+C T_{2}\right)\right\| \leq\left\|C T_{1}\right\|+\left\|C T_{2}\right\|$. The order arrivals and the order quantities will determine the instantly imposed $T T_{k}$ for product $k$. However, different order arrivals will impose an average takt-time $E[T T]$ (Equation (11)), which depends also on the net available work time (WT), i.e. the applied shift regime

$$
T T:=E[T T]=\left.\frac{W T_{\text {avail }}}{\sum_{k} B_{k}}\right|_{\Delta t}
$$


Furthermore, it has to result (from Equation (5a)) written in the Markovian time-dimension that the $C T$ at the bottleneck has to be shorter than the average market-imposed TT (Equation (12))

$$
\forall k, m: C T_{m \mid k} \leq E[T T]
$$

Now we can extend the meaning of $E R$ in Equation (5a), by defining a norm on the ER. The instant $E R$ is given by

$$
\left\|E R_{k}(t)\right\|=\lim _{n \rightarrow 1} \frac{d B_{k}(n)}{d t(n)}=\frac{1}{C T_{k}}=\lambda_{k}(t)
$$

where $d t(n)$ is the time to process one piece. The $E R$ is therefore a specific capacity. Equation (13) is an important statement; it can be seen as the proto-norm from which we can derive different aggregations of $E R$ defining generally a space of nominal normed capacities. The word nominal refers to the standard specific entity-dimension of defined product $E R$.

According to the just enounced Corollary of Balanced Line, in lean JIT pull-manufacturing the $C T_{m}$ can be deterministically established and balanced-out by applying the de-bottlenecking principles, because also the $T T$ is deterministic. The variable TT of Figure 2 is therefore an artificial construct, because it is the consequence of a stochastic OR and hence for each product $k$ different and rather given by the product-specific necessary $C T$ imposed by the equipment. Indeed, in push B \& Q productions, the general, universal equipment usually has a functional-affinity oriented layout (similar equipment grouped into the same department) and a given speed of equipment. This translates according to the product in a resulting specific $C T_{m, k}$ for each product and machine, which usually is not balanced-out. If the affinity is product-oriented we get a pseudo-cell. In the case that the resulting $C T_{m, k}>E[T T]$, the instant capacity is not sufficient to process the workload forming a backlog (BL). And exactly in such cases, the post-optimality conditions come into play for the orders waiting in the backlog (BL) resulting in a backlog waiting time $(B W T)$ defining the solution space of queued orders to comply with OTD.

Now, we are able to define the post-optimality conditions for OTD of an ergodic or non-ergodic multi-product arrival process.

In general, adapting the conditions described in [1] developed for a mono-product, taking the second part of Equation (5), the condition to compute the virtual elasticity for OTD of a backlog (BL) with FIFO-based scheduling principle for a multi-product machine $\mathrm{m}$ becomes

$$
\left\{\begin{array}{l}
B W T_{k-1}(t)+M L T_{k} \leq E D T_{k} \\
E D T_{k}-M L T_{k}=\Delta T_{k}
\end{array}\right.
$$

where $B W T_{k-1}(t)$ is a FIFO-based $\mathrm{BL}$ of maximum $k-1$ products resulting that the $B W T_{k-1}(t)$ at the instant $t$ for product $k$ has to be smaller than the inherent virtual elasticity $\Delta T_{k}$ to meet the OTD requirement of the order for product $k$, i.e.

$$
B W T_{k-1}(t) \leq \Delta T_{k}
$$


Equation (14) represents a necessary condition of product $k$ to be on time. Equation (15) is the synthesized expression of Equation (14) and represents generally the post-optimality condition for OTD compliance, and is an extended interpretation of Equation (5b). If Equation (15) is not satisfied when respecting the FIFO scheduling principle, the OTD condition might be achieved by rescheduling the $\mathrm{BL}$ for product $k$ according to a priority scheduling principle. Therefore, we can now enounce the generalization of the OTD Theorem:

\section{Third Corollary to the Theorem of General Production Requirements (Corollary of Post-optimal Backlog Waiting Time):}

Given is a B \& Q push-based single workstation or a multi-workstation process. The necessary, but not sufficient condition to supply an arrival-order $\Omega_{k}$ for product $k$ with batch-size $B_{k}$ complying with OTD can be generalized that the $B W T_{k-1}$ has to be shorter than the inherent virtual elasticity $\Delta T_{k}$ of the manufacturing system reflecting the post-optimality range of the solution.

Please note, this is only the necessary condition for OTD. If the capacity requirement of Equation (5) is not given, only a weak solution is possible. To have a strong solution depends again on the capacity, i.e. the $E R$, hence the importance to model the ER. Equations (1) and (2) remain of central importance. The virtual elasticity is defined by the First Corollary to the Theorem of General Production Requirements [1]. The generalization just stated by the third corollary is feasible, because the virtual elasticity $\Delta T$ contains the information of the OTD theorem. In the following, we will therefore put the attention on the BWT and how to model it. Please note that this implies that PLT and MLT remain constant. This can be assured by keeping WIP constant which is generally the case in SPF regimes, but has to be implemented for B \& Q push systems (so-called CONWIP or generic pull systems).

In this paper we will try to analyze the problem to set the post-optimal conditions of maximum (or better to say upper limit) $B W T$ while still observing OTD for mixed-product cells. We will apply the theory-derived findings of [1] to ergodic and non-ergodic order arrival processes to comply with the OTD requirement for mixed-production. It has to be noted again and explicitly that in lean JIT-managed pull production systems the problem of post-optimality does not exist. Indeed, the production is deterministically scheduled based on supermarket replenishment-pull. Nevertheless, the customer-order call-offs most probably are of non-ergodic characteristic. In fact, the supermarket de-couples demand and supply, which have different characteristics. Lean JIT-productions based on pull manufacturing principles are therefore asynchronous production systems and not, as often wrongly stated, synchronous. The TR imposes only the pace, but does not directly control the triggering! However, we will hereafter analyze the consequences of a stochastic $O R$ to the production based on a classic Western push production such as represented by envisaged CPPS, which may continue to base mainly on traditional B \& Q production systems. 


\section{Developing Post-Optimality Conditions for Complying with OTD}

For solving the post-optimality conditions for on-time-delivery (OTD) compliance we have to analyze the specific capacity, i.e. the ER of the process. For that we will distinguish different cases. The simple cases for an arrival order process of a single product (mono-product manufacturing) have been analyzed in [1]. In that case, the arriving orders could have been a single order to produce one product or a single order to produce a batch of identical products, but always the same product. Therefore, the logic is the same, because the products to manufacture are identical and has not to be distinguished, i.e. $X_{k}^{-1}(\tau, t)$ or $T T_{k}$; only the pace of manufacturing at the bottleneck would need to change, but this leads to the same solution space shown in Equations (1) and (2). It has to be remarked that general queuing theory only operates with the unspecified term "lot". The $C T$ may refer to a single piece or a batch, which would correspond in our definition to $M L T$. In such a case, the transfer principle can only be batch-wise. Here, this simplification of non-distinction between single product and batch as well as batch or 1-piece transfer principle is not allowed.

However, in the case of a product-mix the arrival orders $\Omega_{k}$ have associated its type of product as well as the order quantity (batch-size $B_{k}$ of the product $k$ ). In this paper, we will analyze two main cases: The first main case considers a multi-product manufacturing with stable deterministic product-mix resulting from an ergodic stochastic $O R$ process. The second main case-increasing the complexity of the problem-we have still a deterministic product-mix of $O R$, however with a non-ergodic order process. Indeed, only the composition of the product-mix may change, but not the number and type of products of the mix, i.e. we treat the arrival cases of a deterministically established product-mix. Furthermore, we will distinguish the case of a single workstation and the case of several workstations grouped into a cell.

\subsection{The Case of an Ergodic OR Process with Deterministic Product-Mix}

\subsubsection{The Simplest Case of a Single Workstation}

In this first case here, we analyze a single multi-product workstation. In such a case, the matrix of Equation (7) reduces to a row-vector with the deterministic product-specific manufacturing cycle times $C T_{k}$ of the $k$ products on the machine $m$

$$
\left[C T_{k}\right]_{m}=\left|\begin{array}{llll}
C T_{1} & C T_{2} & \cdots & C T_{k}
\end{array}\right|_{m}
$$

We assume that the order batch-size $B_{k}$ for the product $k$ is fix and time-invariant, however we will assume they are not equal, i.e. $B_{A} \neq B_{B} \neq B_{k} \neq B_{k+1}$, and is passed as-is to the shopfloor. This is an important assumption, because we will not use a lean Heijunka-levelled scheduling to eliminate Mura (unevenness of $B_{k}$ ). Furthermore, we assume to have a fixed alternating sequence of the product-mix $(\mathrm{A}, \mathrm{B}, \mathrm{C}, \ldots, \mathrm{A}, \mathrm{B}, \mathrm{C}, \ldots)$, resulting then in $\mathrm{PIT}_{\mathrm{A}}=\mathrm{PIT}_{\mathrm{B}}=P I T_{k}=\chi_{k}^{-1}$ (Figure 3). 


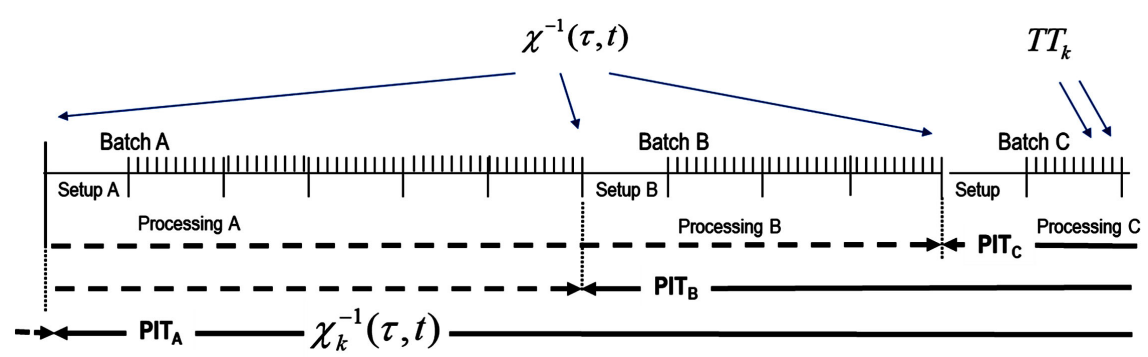

Figure 3. Non-Heijunka pitch-leveled scheduling, however the scheduling is based on a repetitive fixed order of production sequence (with batch $B_{k}$ of different, but fixed size). Then it results in $P I T_{A}=P I T_{B}=P I T_{C}$ independent of $B_{k} ; X^{-1}(\tau, t)$ is the product-independent inter-arrival time of orders. $\chi_{k}^{-1}(\tau, t)$ is the generic product-specific inter-scheduling time of products.

Due to the assumption of deterministic order-mix sequence, it results in this case that for the order inter-arrival time $X_{k}^{-1}(\tau, t)$ Equation (17) applies

$$
\forall k:\left\{\begin{array}{l}
\chi_{k}^{-1}(\tau, t)=\chi_{k}^{-1}=P I T_{k}=P I T_{k+1} \\
\text { and therefore } P I T_{k}=W T T_{m \mid K}
\end{array}\right.
$$

Equation system 17 leads to the important conclusion that $\forall k: \chi_{k}^{-1}(\tau)=W T T_{m, K}$ or better said, they have to be $\forall k: W T T_{m, K}=\chi_{k}^{-1}$.

If the arrival rate $X(\tau, t)$ of $\Omega_{k}$ respects the ordered WTT-composition, then the "between"-sample statistical-parameters of orders in the periodically recurring timeframe $\Delta t=W T T$ do not change and the arrival process of orders $\Omega_{k}$ is ergodic, i.e. $X\left(\tau, t_{1}\right)=X\left(\tau, t_{2}\right)=X(\tau)$ and $X$ would be deterministic. Furthermore, the $X(\tau, t)$ of Figure 3 is the product-independent arrival rate of the single product arrival rates $X_{k}(\tau, t)$, which symbolically we can represent as

$$
X(\tau, t)=\sum_{k} X_{k}\left(\tau_{k}, t\right)
$$

and specifically also (if $P I T_{k} \neq P I T_{k+1}$ )

$$
\chi^{-1}(\tau)=\sum_{k} \chi_{k}^{-1}
$$

The resulting average performance, intended as nominal $E R$ of a multi-product workstation $\mathrm{m}$, is given by Equation (18), which reflects the real average $E R$ performance of the workstation in the timespan $W T T$. We can call it nominal, because characteristic of the cell. In this case the $W T T$ product/cell characteristic is invariant. Equation (18) defines a norm on the capacity, i.e. the physical ER of a multi-product equipment

$$
\left\|E\left[E R_{m}(\Delta t)\right]\right\|=\left.\frac{\sum_{k} B_{k}}{W T T_{m \mid K}}\right|_{\Delta t=W T T}=\lambda_{m}(W T T)
$$

The nominal ER of the multi-product workstation in Equation (18) assumes to produce repeatedly the same product mix with the same $\chi_{k}^{-1}$ for all products $k$. If the ergodicity in $\Delta t$ periods of $X^{-1}(\tau)$ does not apply, Equation (18) is not valid and has to be adapted (see the next main section). However, the instant 
$E R$ according to Equation (13) is always valid. Equation (18) is a nominal productivity characteristic of a machine with a given product-mix. It is necessary to distinguish the average nominal exit rate $\lambda_{m}(W T T)$ in Equation (18) of a workstation, which we could apply for a rescheduled ergodic $I R$ process in discrete timespan, from the instant exit rate of product $k$ (Equation (13)) represented by $\lambda_{k}(t)$ on the workstation. The distinction of nominal (which for a multi-product machine is an average) and instant ER is also applicable in the contingent situation of modelling deterministic specific order arrivals to reduce complexity of infinitesimal representation. In the following, we will try to give an algebraic representation and solution to a dynamic system.

Furthermore, to calculate $M L T$ we can rely on Equation (4). For a single workstation the distinction of SPF and B \& Q vanishes and the $M L T$ can be computed as

$$
M L T_{k}=B_{k} \cdot C T_{k}=\frac{B_{k}}{\lambda_{k}}
$$

which reflects Little's law for a single workstation, but at which has to be added the set-up time $(S T)$. With the assumed fixed sequence of arrivals, the problem situation becomes deterministic. If the batch-size of the arriving orders is too unbalanced, i.e. a successive series of large batches, an alternation of scheduling large and small batches may help to become OTD compliant for all orders. However, arrival orders cannot always be influenced if a FIFO order processing is supposed. The $B W T$ can be modelled generally by setting $E R_{k}=\lambda_{k}$ resulting

$$
B W T \leq \frac{B L}{E R} \rightarrow B W T_{k-1}=\sum_{k=2}^{K-1}\left(S T_{k}+\frac{B_{k}}{\lambda_{k}}\right)+\frac{B_{1}-\lambda_{1} \cdot \Delta t}{\lambda_{1}}
$$

The $B W T$ of Equation (19) is composed of a "waiting" $B L$ and a $B L$ being currently in the course to be "worked down" usually called WIP where $\Delta t$ is the time already elapsed the product $k=1$ being manufactured. In a FIFO organized waiting queue, order $\Omega_{k}$ for product $k$ has to wait until the $B L$ is worked-down. Notice that the $B L$-waiting term of Equation (19) shows only apparent similarity to Equation (1): indeed, Equation (1) is a $O R / E R$ comparison during a certain time-span whereas Equation (19) is a $B L / E R$ ratio computing the time needed to work down a $B L$. Please note that $X_{k}$ are orders per time-unit; however $\lambda_{k}$ are pieces per time-unit. A piece-specific "takt" would be given by $O R_{k}=B_{k} \cdot X_{k}$. However, if the order frequency $X_{k}$ of $\Omega_{k}$ is too high, the $B W T_{k-1}$ may exceed the allowed $\Delta T_{k}$ of $\Omega_{k}$ to be supplied on time. Instead of applying the extended and analytical $B W T$ according to Equation (19), we could use the nominal $E R$ according to Equation (18) approximating

$$
B W T_{k-1} \approx \frac{\sum_{k=1}^{K-1} B_{k}}{\lambda_{m}(W T T)}
$$

\subsubsection{The Case of a Manufacturing Cell with Several Workstations}

In this sub-section, we will analyze the post-optimality for OTD compliance for 
an order arrival process with a defined product-mix manufactured within a single manufacturing cell or transfer line (TFL) according to the matrix of Equation (8). We will omit in future "or TFL", being ideally a cell a TFL process in miniature. A manufacturing cell is the physical materialization of a process, which is a sequence of several logical steps (manual operations at workstations or machining operations on a workstation). The situation to be analyzed is described in Figure 4.

In addition, we will suppose that a SPF is applied to the process. Indeed, according to the just before enounced Corollary of Balanced Line by applying the de-bottleneck principles, it is generally possible to transform an unbalanced SPF with unequal $C T$ (SP handling) into a perfectly balanced SPF with operations of equal $C T$. This allows calculating the $M L T$ according to Equation (4). We will therefore analyze the case for a balanced SPF. Applying mathematical transformation by setting $m \cdot C T_{k}=W C_{k}$ it is possible to write Equation (4) as

$$
M L T_{k}^{S P F}=\left(B_{k}-1\right) \cdot C T_{k}+W C_{k}
$$

reflecting that $M L T$ depends on the batch-size, cycle time, and work content. This shows very well, that it is not sufficient to reduce cycle times, and therefore also the work content, but also batch-size has to be reduced to shorten lead-time. And exactly this is the aim of pitch-levelled Heijunka-box of the TPS to reduce Mura (unevenness). If the sequenced product-mix of orders (the scheduling order of the $k$ products) remains stable in the time-dimension $t$, we have a perfect ergodic process. The instant $E R_{k}(t)$ of the cell for product $k$, which corresponds to the $1 / C T_{m k}$ of the cell or generally to the bottleneck workstation according to Equation (13), is valid also for a manufacturing cell. Due to the fact, that the products of a perfectly conceived cell follow the Linear Dependency Theorem, the bottleneck workstation is time-invariant. Indeed, due to the linear dependency theorem the First Corollary to the WTT Aggregated Bottleneck Theorem, which states that the bottleneck becomes time-variant in a mixed-product manufacturing cell, should not materialize in a lean conceived JIT cell. However, this will not be the case in a CPPS for a B \& Q manufacturing system. The bottleneck-workstation of a multi-product multi-workstation cell (mixed-model) is defined by the before mentioned Theorem of Generalized Throughput (or

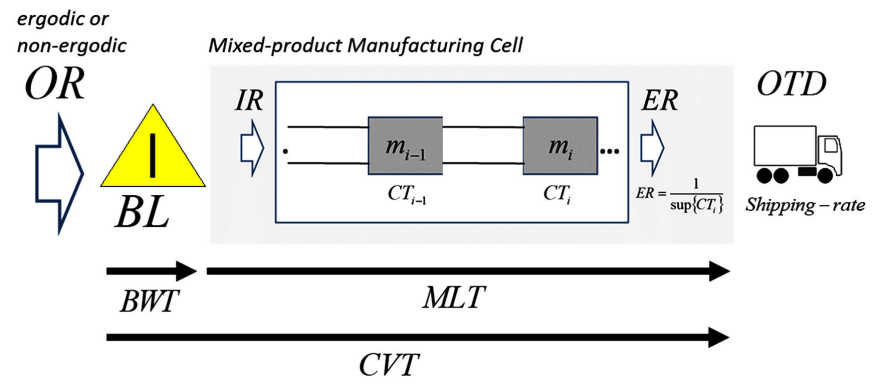

Figure 4. General representation of a FIFO push-scheduled, but flow-based manufacturing cell applying 1-piece transfer principle with $\mathrm{m}$ workstations manufacturing $k$ products reflected by Equation (8) (adapted from [1]). 
WTT-aggregated Bottleneck Theorem). This translates into an average exit rate $E\left[E R_{k}(t)\right]$ of the cell for the $k$ products, representing the mid-term production capacity of the manufacturing cell, and therefore, depends on the $W T T_{m K}$ of the bottleneck machine, i.e.

$$
E\left[E R_{k}(t)\right]_{\text {cell }}=E\left[\lambda_{\Delta t}\right]_{\substack{\text { sup }\left\{W T T_{m \mid K}\right\} \\ \text { s. }}}
$$

Also in this case of increased complexity, the average exit rate $E[\lambda]$ of the cell will correspond to the ER of the bottleneck machine $\lambda_{m}$ which at the instant $\mathrm{t}$ is determined by the universally valid Theorem of Throughput (Bottleneck Theorem). The average exit rate of the machine $E\left[\lambda_{m}\right]$ can be increased by reducing setup-time $(S T)$ applying lean SMED technique (single minute exchange of die), because $W T T$ contains the $S T$. However, to implement a perfect balanced SPF, the Tayloristic-splitting of $C T$ would lead to an additional workstation, de-bottlenecking technique to be used in a constraint process, increasing the column dimension $m$ of matrix of Equation (8).

Now, based on the nominal ER defined in Equation (18) for a single workstation, we can extend the departure rate norm applied to a manufacturing cell $j$. The nominal capacity of the cell results in Equation (20)

$$
\left\|E\left[E R_{j}(\Delta t)\right]\right\|=\left.\frac{\sum_{k} B_{k}}{C T T_{j \mid K}}\right|_{\Delta t=C T T}=\lambda_{j}(C T T)
$$

where the cell turnover time (CTT) is similar to the $W T T$ defined in Equation (3) and is the time to produce once the product-mix manufactured within the cell. The $C T T$ allows defining within a complex multi-cell manufacturing system the bottleneck cell. The $C T T$ is defined in Equation (21) where $K$ denotes the number of different products manufactured in the cell $j$ with $m$ machines composing the cell. For further information consult [2].

$$
C T T_{j \mid K}=\sum_{k}\left(\sum_{m} S T_{m \mid k}+M L T_{k}\right)
$$

Please note that in a multi-station cell not the process lead-time PLT of a single product applies, but the $M L T$ of the entire batch. Furthermore, the improvement potential exists to shorten the sum of $S T$ starting already when the last piece of the batch has left the first workstation (during emptying set-up), or by parallelizing the $S T$. This leads directly to define an additional implementation principle: the Set-up Principle (after emptying set-up, during emptying set-up). To facilitate calculation, we will assume after emptying set-up principle (ST and MLT do not overlap, i.e. $\sum_{m} S T_{m, k+1} \cap M L T_{k}=\varnothing$ ).

The post-optimal condition for the last order arrival $\Omega_{k}$ for a cell with $\mathrm{M}$ machines (workstations) has to obey to the virtual elasticity Equation (15) where BWT can be approximated to Equation (22), which is similar to Equation (19)

$$
B W T \leq \frac{B L}{E R} \rightarrow B W T_{k-1}(t)=\sum_{k=2}^{K-1}\left(\sum_{m=1}^{M} S T_{m \mid k}+M L T_{k}\right)+m l t_{1}(t)
$$

where the lower case writing of $M L T$ shows that the WIP status of the process 
progress depends on the instant $t$ and therefore $m t_{1}(t)<M L T_{1}$ results depending on the arrival-instant of the order $\Omega_{k}$. The index $k$ represents here the orders of products and not the number of products defining the mix. We leave to the interested reader to develop the analytic expression of mlt $t$ ). Again, Equation (22) is an approximation; indeed, the next order may not wait until the last piece of the batch has left the last operation, but will enter the cell as soon as the last piece has left the first operation and the set-up of the first machine has been completed (during emptying set-up principle). This is less important in manually operated cells than in automatic high performance FMS TFL.

\subsection{The Case of a Non-Ergodic OR Process with Deterministic Product-Mix}

\subsubsection{The Case of a Single Multi-Product Workstation}

The before analyzed cases of product k-ordered arrival and input-sequence of $\Omega_{k}$ is an artificial assumption for non-JIT productions. Now in this case we analyze the more realistic situation of random arrival of orders $\Omega_{k}(t)$ of the different products of non-ergodic characteristic. However, we will maintain the fixed batch-size for every specific product of the still deterministic product-mix. This simple assumption at least allows to maintaining the important concept of WTT also for non Heijunka-levelled cells (or single workstations). Nevertheless, if the virtual elasticity $\Delta T$ is large enough (i.e. $\Delta T>W T T$ ) the possibility exists to reschedule the random order entry by sequencing the input rate $(I R)$ into the shopfloor according to the $W T T$ composition to approach an ergodic process for the IR for timeframes $\Delta T>W T T$. This important property leads directly to enounce a new corollary

Forth Corollary to the Theorem of General Production Requirements (Corollary of Ergodic Backlog Rescheduling):

Given is a non-ergodic $O R$ with a deterministic product-mix manufacturing. If the virtual elasticity $\Delta T$ is large enough $(\Delta T>W T T)$, in certain cases the possibility exists to transform the non-ergodic $O R$ in an ergodic $I R$ with a defined scheduling sequence, trying at least to approach perfect ergodicity.

However, the resulting rescheduled $I R$ may constitute only a weak solution for OTD compliance. The conditions to have a strong solution is given by the tautological Equation (23)

$$
\lim _{\Delta T_{k} \gg W T T} \frac{\Delta T_{k}}{W T T}=\infty
$$

On the other hand, the ergodic process characteristic is important in order to have an ergodic output performance, i.e. to approaching a deterministic performance according to the nominal ER of Equation (18). Furthermore, the deterministic product-mix allows to keeping the CT-vector representation of Equation (16). This will not be the case in CPPS with stochastic product-mix, because the $C T$-array cannot be defined in advance. The rescheduling to obtain an er- 
godic process corresponds to the concept of Toyota's Mura reduction. Now, for the $B W T$ of Equation (19), the index $k-1$ is the rescheduled order of the $I R$. The verification for OTD has to be done for each product in the $B L$ to satisfy Equation (15). As the forth corollary states, we are in presence of a weak solution, because it may suit only for some product-orders. In this case a further rescheduling may be applied although loosing ergodicity. In that case, the nominal $E R$ of Equation (18) is not valid anymore, as this is not representing adequately the actual system status.

Generally, we have seen that in the case of a deterministic product-mix the ergodicity for the $O R$ is not required, because the IR can be rescheduled if $\Delta t$ is large enough. Under these assumptions, it would not be necessary to further analyze the $O R$ characteristic and its repercussion on the ER and OTD. However, we will analyze it in view of non-ergodic and non-deterministic product-mix performance, in the case that the just enounced Corollary of Ergodic Backlog Rescheduling may not apply for the $I R$, because we have a non-deterministic product-mix.

In that case, the assumptions made in Equation (17) for the $O R$ are not valid anymore, because the concept of PIT (deterministic interval) has definitely to be substituted with $X_{k}^{-1}(\tau, t)$ based on a stochastically variable interval. These circumstances lead to Equation (24)

$$
\forall k: P I T_{k}\left(t_{1}\right) \neq P I T_{k}\left(t_{2}\right) \Rightarrow X_{k}^{-1}(\tau, t)
$$

and therefore $P I T_{k} \neq W T T_{m, K}$ as well as $X_{k}^{-1}(\tau, t) \neq X_{k+1}^{-1}(\tau, t)$

We are in presence of a non-ergodic arrival order process $\Omega_{k}(t)$, or $I R$, when Equation (25) applies

$$
\forall k: X_{k}^{-1}\left(\tau, \Delta t_{1}\right) \neq X_{k}^{-1}\left(\tau, \Delta t_{2}\right)
$$

i.e. the inter-arrival times $\tau$ show a non-constant distribution in the time dimension $t$. If we apply the concept of norm on the capacity defined in Equation (18) to a single multi-product workstation with a deterministic product-mix, but stochastic arrival of orders $\Omega_{k}(t)$ the situation gets complicated. In fact, we cannot talk anymore about a nominal capacity of the workstation according to Equation (18), because the real $E R$ of the workstation becomes highly non-ergodic itself, which we could represent as (Equation (26))

$$
\left\|E\left[E R_{m}(\Delta t)\right]\right\|=\left.\frac{\sum_{k} \Omega_{k}\left(B_{k}, t\right)}{W T T_{m \mid K}}\right|_{\Delta t=W T T}=\lambda_{m}^{*}\left(\Omega_{k}, W T T\right)
$$

Nevertheless, due to the non-ergodicity it is necessary, and thanks to the WTT, it is possible to talk of a pseudo-nominal ER (or pseudo-mean capacity) $\lambda_{m}^{*}\left(\Omega_{k}, W T T\right)$ due to the presence of the WTT in the denominator of Equation (26) whereas the batches $B_{k}$ of different products $k$ in the nominator depend on the orders $\Omega_{k}(t)$ in the time period $\Delta t=W T T$. If compared to Equation (18), Equation (26) allows assessing the nominal gain or loss in capacity due to mix variation. The real, stochastic changing, mean $E R$ is shown in Equation (27), 
which has also the real orders $\Omega_{k}(t)$ in the denominator during the considered timespan $\Delta t$; the $E R$ itself in the subsequent timespans $\Delta t$ becomes non-ergodic and is a function of $B_{k}$ and $t$

$$
\left\|E\left[E R_{m}(\Delta t)\right]\right\|=\left.\frac{\sum_{k} \Omega_{k}\left(B_{k}, t\right)}{\sum_{k} \Omega_{k}\left(S T_{k}+C T_{k} \cdot B_{k}\right)}\right|_{\Delta t}=\bar{\lambda}_{m}\left(\Omega_{k}, \Delta t\right)
$$

The real $E R$ in the instant $t_{k}$ of the order $\Omega_{k}(t)$ is determined according to Equation (13) by the $C T_{k}$ of the product $k$ (Equation (28))

$$
E\left[E R_{m}\left(\Omega_{k}\right)\right]_{t_{k}}=\lambda_{m}\left(\Omega_{k}\right)=\frac{1}{C T_{k}}
$$

We can therefore talk about an instant real $E R$ (Equation (28)) which reflects Equation (13), an average real $E R$ (Equation (27)), and a pseudo-nominal ER (Equation (26)). The nominal ER according to Equation (18) exists also for non-ergodic processes, but will realistically not materialize. As already stated, the pseudo-nominal ER of Equation (26) (with equal timespan corresponding to $W T T$ ) could be used to evaluate comparatively the $E R$ of the workstation for several instants based on the actually produced product-mix.

Now, to be OTD compliant, Equation (15) has to be satisfied in any case, which is generally valid also for non-ergodic arrival processes. Due to the application of the product-specific $E R_{k}$, which is $\lambda_{k}$, Equation (19) is independent of the ergodic process characteristics and, therefore, is also valid in the case of non-ergodic processes.

\subsubsection{The Case of a Manufacturing Cell}

Also for a deterministic product-mix manufacturing cell, defined according to Equation (8) and represented by Figure 4, the just enounced 4th Corollary to the Theorem of General Production Requirements (Corollary of Ergodic Backlog Rescheduling) applies. However, as we have just seen, the ergodicity is not necessary to comply with Equation (15), because Equation (15) has to be satisfied for all processes in order to be on time. Therefore, the post-optimal condition for OTD compliance of an order $\Omega_{k}$ is also given for a non-ergodic arrival process (order intake) by Equation (22). We can therefore enounce the

Lemma to the Third Corollary of the Theorem of General Production Requirements (Lemma to the Corollary of Post-optimal BWT), the Lemma of Ergodic-independent Validity:

The necessary condition $B W T_{k-1}(t) \leq \Delta T_{k}$ for OTD is generally valid, independent of the character of order arrivals, i.e. deterministic, ergodic, or non-ergodic.

The equivalent $E R$ to Equation (26) ( $E R$ valid for a single workstation) for a multi-product manufacturing cell $\mathrm{j}$ is

$$
\left\|E\left[E R_{j}(t)\right]_{\Delta t}\right\|=\left.\frac{\sum_{k} \Omega_{k}\left(B_{k}, t\right)}{C T T_{j \mid k}}\right|_{\Delta t=C T T}=\lambda_{j}^{*}\left(\Omega_{k}, C T T\right)
$$


Equation (29) gives the pseudo-nominal ER of the cell. We call it "nominal" due to the presence of the CTT in the denominator, and "pseudo", because in the nominator may not appear the corresponding batches of the CTT product-mix in the timeframe $C T T$. It is highly improbable to have in a non-ergodic order intake regime the materialization of the nominal ER, but not impossible. Due to the application of the just enounced Corollary of Ergodic BL Rescheduling, the possibility exists of rescheduling the orders for shopfloor release if the virtual elasticity is high enough approaching Equation (23). The desired characteristic of ergodicity translates into Toyota's Mura, i.e. reducing unevenness.

However, the average real $E R$ of the cell in the generic time span $\Delta t$ derived from Equation (27) is given by the real orders also in the denominator leading to Equation (30)

$$
\begin{aligned}
\left\|E\left[E R_{j}(t)\right]_{\Delta t}\right\| & =\left.\frac{\sum_{k} \Omega_{k}\left(B_{k}, t\right)}{\sum_{k} \Omega_{k}\left(M L T_{k}\right)}\right|_{\Delta t}=\left.\frac{\sum_{k} B_{k}(n)}{\sum_{k}\left(\sum_{m} S T_{m \mid k}+M L T_{k}\right)}\right|_{\Delta t} \\
& =\bar{\lambda}_{j}\left(\Omega_{k}, \Delta t\right)
\end{aligned}
$$

From the denominator it is evident that Equation (30) implies the after emptying set-up principle to change the set-up of the workstations (machines) from product batch $k$ to $k+1$ where $\mathrm{m}$ are the machines in the cell. The $M L T$ is calculated according to Equation (4) for a SPF. However, Equation (30) is generally valid and therefore also for an entire batch transfer principle, or for a n-piece transfer principle with obvious different calculation of $M L T$ [2]. Please note that an unfortunate mix-shifted product-mix may deteriorate the intrinsic nominal $E R$ of the cell reducing the profitability potential, or increasing the profitability in the case of a favorable mix-shift. The key parameter is therefore not the $E R$ in terms of manufactured pieces, but the generated margin contribution (MC) per time unit. Indeed, the industrial logic has changed from MC per piece to MC per time. However, this is another topic.

With the definition of normed $E R$ we can now generalize the first condition 5 a of Equation (5) by setting

$$
\left\|E\left[E R_{j}\left(\Omega_{k}\right)\right]_{\Delta t}\right\| \geq E\left[\operatorname{OR}\left(\Omega_{k}\right)\right]_{\Delta t}
$$

Equation (31) is generally valid and can be rewritten by emphasizing the distribution of orders as

$$
\left\|E\left[\lambda_{j}\left(\chi_{k}\right)\right]_{\Delta t}\right\| \geq E\left[X_{k}(\tau, t)\right]_{\Delta t}
$$

If the necessary condition of Equation (15), i.e. $B W T_{k-1}(t) \leq \Delta T_{k}$, is respected, then Equation (32) stresses the distribution of arrivals and the capacity requirement for OTD compliance. For a strong solution, Equation (32) is subject to the Second Corollary to the Theorem of General Production Requirements (Corollary of Strong and Weak OTD Solution) regarding the distribution of arrivals [1]. 


\section{Outlook for Complex Manufacturing Systems}

A complex manufacturing system (multi cell manufacturing) is a production system where different products are manufactured in several manufacturing cells or dedicated shopfloor departments (jobshops) producing a complex component or a final product. The multi-product cells may constitute different stages of the manufacturing process with increasing value-add content supplying a backbone TFL or being part of a graph-based organized shopfloor. We can therefore distinguish between a product-structured inter-linked cellular manufacturing and a manufacturing with a generic complex layout.

For the inter-linked cellular manufacturing there is no further complication due to the presence of Kanban-managed supermarkets decoupling the various manufacturing cells. The manufacturing cells are managed autonomously solely triggered by the withdrawals of the products (components) out of the supermarket. The backlog is limited by the production Kanbans in the Heijunka levelling box. The supermarket is a proof that lean JIT manufacturing is not a synchronous system-it bases on asynchrony by decoupling supply from demand. However, JIS manufacturing systems are synchronous systems where the supply-cell is synchronized with the final assembly line (demand scheduled). By the way, the logic of Toyota's complexity reducing cell-fractional manufacturing has already been applied long time before by Roman emperors to govern complex territorial systems known as "divide et impera". Therefore, there is no need to further analyze the dynamic of lean JIT manufacturing systems.

However, a graph-modelled complex shopfloor which manufactures not only a single component, but a finished product has the task to control autonomously interacting, intelligent single objects (workstations, AGVs, components) along the whole flexible manufacturing path. Further challenges may arise regarding performance in terms of ER, MLT, and OTD in non-optimally conceived CPPS, trying to solve the quest of the optimal manufacturing process in its entirety. Nevertheless, the new Lemma of Ergodic-independent Validity extends the applicability of the Corollary of Post-optimal BWT also to CPPS; this will be very important for OTD dynamic rescheduling. In addition, the here defined norm on the ER will allow the analysis of the performance of CPPS with its high non-ergodic commercial order characteristic. The OTD corollaries stand at the base of the predominantly B \& Q driven CPPS trying to comply to customer OTD. JIT manufacturing systems guarantee intrinsically OTD, hence the superiority of the cell-fractional "divide et impera"-model allowing pull-JIT manufacturing. This tricky, but interesting topic of CPPS will be addressed in a later paper.

\section{Conclusion}

We have seen that the traditional Western large B \& Q manufacturing combined with OTIF deliveries can compromise the OTD requirement for other products. However, the TPS JIT pitch-based pull-manufacturing has solved this problem 
for a deterministic product-mix. In this paper, we set the necessary and sufficient conditions to generalize the OTD theorem for a deterministic product-mix. The Third Corollary to the Theorem of General Production Requirements (Corollary of Post-optimal BWT) giving only the necessary, but not sufficient condition for OTD has induced to define a normed ER to derive also the sufficiency condition for OTD in different production regimes. The norm has been defined for various "objects" (such as single workstations or manufacturing cells in presence of ergodic and non-ergodic order regimes) resulting in a space of normed nominal capacities which completes the Corollary of Post-optimal BWT with the sufficiency condition for OTD according to Equation (5). This allows structuring the generic manufacturing term ER (called departure rate in queuing theory). These findings are important, because gaining a rational law-based Cartesian insight into the theory of general OTD requirements for queued manufacturing systems also in view of future CPPS, allowing limitless product customization. As demonstrated, the virtual elasticity applies also for a mixed-product manufacturing with deterministic product-mix, independent of the ergodic order-arrival characteristic as the new enounced Forth Corollary to the Theorem of General Production Requirements (Corollary of Ergodic BL Rescheduling) states. However, if OR processes are highly non-ergodic and show a non-deterministic product-mix, such as envisaged by Industry 4.0-type CPPS, the findings such as the new enounced Corollary of Ergodic BL Rescheduling as well as other conclusions elaborated in this paper may not be applicable and may need to be revised. This has to be further investigated. Nevertheless, the result of this paper gives insights into the manufacturing "physics" in presence of different commercial order-arrival characteristics and shopfloor implementation principles. It allows understanding the behavior and calculating the performance of a simple cell-type manufacturing system without computer simulation. And finally, the hypothesis of extended conditions for general OTD compliance has been proven in a deterministic product-mix push manufacturing environment.

\section{Conflicts of Interest}

The authors declare no conflicts of interest regarding the publication of this paper.

\section{References}

[1] Rüttimann, B.G. and Stöckli, M.T. (2020) Exploiting Virtual Elasticity of Production Systems to Respecting OTD-Part 1: Post-Optimality Conditions for Ergodic Order Arrivals in Fixed Capacity Regimes. AJOR, 10, 321-342.

https://doi.org/10.4236/ajor.2020.106018

[2] Rüttimann, B.G. (2017) Lean Compendium-Introduction to Modern Manufacturing Theory. Springer, Berlin. https://doi.org/10.1007/978-3-319-58601-4

[3] Rüttimann, B.G. (2015) Discourse about Linear Programming and Lean Manufacturing: Two Different Approaches with a Similar Converging Rational. JSSM, 8, 85-91. https://doi.org/10.4236/jssm.2015.81010 